\title{
INCIDENCE OF ORONASOPHARYNGEAL \& LARYNGEAL TUBERCULOSIS CASES IN PULMONARY TUBERCULOSIS CASES
}

\author{
Authors: Dr. Mohd Aftab (1), Dr. Sachin Jain (2), Dr. Pankaj Kumar Tiwari (3), Dr Ram Manohar Verma \\ (4), Dr. Daya Shanker (5).
}

Authors Affiliations: (1)Associate Professor, (2) Professor and Head, (3) Junior Resident, (4) Junior Resident, (5) Junior Resident, Department of ENT \& Head Neck Surgery, M.L.N. Medical College, Prayagraj, Uttar Pradesh, India.

\section{ABSTRACT}

Background: Tuberculosis is a major global health problem. Tuberculosis may be Pulmonary or Extrapulmonary. Oro-nasopharyngeal and Laryngeal tuberculosis are a form of Extrapulmonary Tuberculosis. Nasopharyngeal tuberculosis presents with neck mass, nasal obstruction, rhinorrhoea, epistaxis, Otalgia, hearing loss and cervical Lymphadenopathy. Common symptom of Laryngeal tuberculosis is hoarseness of voice. This study will increase awareness regarding Oro-nasopharyngeal / Laryngeal tuberculosis and will help in differentiating it from Oro-nasopharyngeal / Laryngeal carcinoma.

Objective: To find the incidence of Oronasopharyngeal and Laryngeal Tuberculosis among patients of PulmonaryTuberculosis.

Method: A total of 80 patients of Pulmonary Tuberculosis were selected and investigated for symptoms of Oro-nasopharyngeal and Laryngeal tuberculosis.

Results: Study revealed that $1.25 \%$ (1 patient out of 80 ) of total study subjects had Nasopharyngeal tuberculosis.

Conclusion: The study showed that the incidence of Oro-nasopharyngeal and Laryngeal tuberculosis among patients of PulmonaryTuberculosis was very less.

Keywords: Oro-nasopharyngeal, Laryngeal Tuberculosis, Pulmonary Tuberculosis,
ExtrapulmonaryTuberculosis.

\section{INTRODUCTION}

Tuberculosis (TB) is a major global health problem. It causes ill-health for approximately 10 million people each year and is one of the top ten causes of death worldwide. Tuberculosis has remained one of the world's deadliest communicable disease. ${ }^{\prime}$

Tuberculosis is the ninth leading cause of death worldwide and the leading cause from a single infectious agent, ranking above HIV/ AIDS. In 2016, globally there were as estimated 1.3 million Tuberculosis deaths among HIVnegative people (down from 1.7 million in 2000) and an additional 374000 deaths among HIV positive people.

No part of human body is immune to tuberculosis; it can affect the lung (Pulmonary tuberculosis) \& other sites (Extra pulmonary tuberculosis). Approximately $25 \%$ of case are extra pulmonary tuberculosis and of which head $\&$ Neck regions can be affected in up to $10 \%$ of these case with cervical lymph nodes most commonly involved. ${ }^{2}$ Tuberculosis of larynx was often misdiagnosed as carcinoma of larynx in patients with hoarseness and weight loss. It also presents with productive cough, pulmonary rales, and absence of cervical lymphadenopathy, which is commonly found in laryngeal carcinoma. Tuberculosis laryngitis is the most infectious form of disease, thorough physical examination and chest 
roentgenoraphic study should precede laryngoscopic examination of patients in whom tuberculosis may seem to be carcinoma of larynx. ${ }^{2}$

Tuberculosis can involve the nasopharynx primarily without affecting any other system termed a rare condition as nasopharyngeal tuberculosis or secondary to pulmonary or extrapulmonary involvement, when the portal of entry is upper respiratory tract and the route of spread is via hematogenous or lymphatic system.Majority of patients of nasopharyngeal tuberculosis presents with neck mass, nasal obstruction, rhinorrhoea, epistaxis, otalgiaor hearing loss, constitutional symptoms occurs in $12-30 \%$ cases of nasopharyngeal tuberculosis. ${ }^{3}$

However, nasopharyngeal tuberculosis is usually ignored because it has varied clinical manifestations and similar presentation with other common head and neck diseases. Nasopharyngeal tuberculosis may mimic nasopharyngeal carcinoma, both can present with cervical lymphadenopathy, nasal discharge, or nasal obstruction cervical lymphadenopathy together with the nasopharyngeal symptoms related to the mass or mucosal irregularity, makes the differential diagnosis from carcinoma difficult. Nasopharyngeal tuberculosis can co-exist with other conditions like laryngeal tuberculosis or oropharyngeal tuberculosis.

The diagnosis of oronasopharyngeal and laryngeal tuberculosis is often difficult and missed due to overlapping manifestations with carcinomas and other infections. This study was conducted to find out the incidence of Oronasopharyngeal and Laryngeal tuberculosis in Pulmonary tuberculosis cases. It will help in assessing the disease burden of oronasopharyngeal and laryngeal tuberculosis.

\section{MATERIALS AND METHODS}

This retroprospective study was conducted in S.R.N.Hospital, Prayagraj and TejBahadurSapru tuberculosis Hospital, Prayagraj. Patients were taken from duration (August 2018 to August 2019).This study was conducted after due clearance from institutional ethics committee. Patients were properly informed and written informed consent were taken from patients before participating in the study in an approved format. Patients selected for study were properly examined and history of contact, family history and duration of symptoms was taken and these patients were examined by Anterior rhinoscopy, diagnostic nasal endoscopy and direct fibreoptic laryngoscopy. Throat swab of patients for study were sent for bacteriological examination by (ZiehlNeelsen stain or BACTEC culture) and sputum examination by (Light Emission Diode Fluorescence Microscopy). Patients who were found positive for Acid Fast Bacilli from throat swab culture examination, were confirmed by histopathological examination of biopsied materials. For retrospective patients, case sheets of patients were collected from record keeping section, their addresses and phone number were obtained and called by letters and phones. After that their detailed history (family history, duration of symptoms) and investigations by which they diagnosed were taken.

\section{RESULTS}

This study was conducted on 80 patients and out of them distribution on the basis of chief complaints is as follows - 
Table 1 - Distribution of Study subjects according to chief complaints.

\begin{tabular}{|l|c|c|}
\hline \multirow{2}{*}{ Chief Complaints } & \multicolumn{2}{c|}{ Study Subjects } \\
\cline { 2 - 3 } & No. & $\%$ \\
\hline Cough \& Expectoration & 49 & $61.3 \%$ \\
\hline Breathlessness & 39 & $48.8 \%$ \\
\hline Chest Pain & 1 & $1.3 \%$ \\
\hline Haemoptysis & 8 & $10.0 \%$ \\
\hline Epistaxis & 1 & $1.3 \%$ \\
\hline Sore throat & 1 & $1.3 \%$ \\
\hline Cervical Swelling & 10 & $12.5 \%$ \\
\hline Change in Voice & 1 & $1.3 \%$ \\
\hline
\end{tabular}

Table-1 shows that maximum number of subjects (61.3\%) complained of cough and expectoration, less than half $(48.8 \%)$ complained of breathlessness, some of the subjects $(12.5 \%)$ complained of cervical swelling and nearly the same proportion (10.0\%) mentioned haemoptysis. Very few of the subjects $(1.3 \%)$ complained about chest pain, epistaxis, sore throat or change in voice.

Table 2 - Findings on Anterior Rhinoscopy

\begin{tabular}{|l|c|c|}
\hline \multirow{2}{*}{ Findings on Anterior Rhinoscopy } & \multicolumn{2}{|c|}{ Study Subjects } \\
\cline { 2 - 3 } & No. & $\%$ \\
\hline Change in nasal mucosa & 4 & $5.0 \%$ \\
\hline Nasal Discharge & 3 & $3.8 \%$ \\
\hline Turbinate Hypertrophy & 1 & $1.3 \%$ \\
\hline Deviated Nasal Septum & 7 & $8.8 \%$ \\
\hline No abnormality detected & 65 & $81.25 \%$ \\
\hline
\end{tabular}

Table-2 shows that maximum (81.25\%) of study subjects were found with no abnormality on anterior rhinoscopy, $8.8 \%$ of study subjects were found to have deviated nasal septum, 3.8\% had nasal discharge, $5.0 \%$ had changes in nasal mucosa while only $1.3 \%$ had hypertrophied turbinates.

Table 3 - Findings on Diagnostic Nasal Endoscopy

\begin{tabular}{|l|c|c|}
\hline \multirow{2}{*}{ Findings on Diagnostic Nasal Endoscopy } & \multicolumn{2}{|c|}{ Study Subjects } \\
\cline { 2 - 3 } & No. & $\%$ \\
\hline Changes in mucosa of nasopharynx & 5 & $6.3 \%$ \\
\hline Eustachian tube - not patent & 10 & $12.5 \%$ \\
\hline Thickened lateral and posterior wall of nasopharynx & 3 & $3.8 \%$ \\
\hline No abnormality detected & 62 & $77.5 \%$ \\
\hline
\end{tabular}

Table-3 shows that maximum (77.5\%) of study subjects were found with no abnormality on diagnostic nasal endoscopy, $12.5 \%$ of subjects had Eustachian tube not patent, $6.3 \%$ had changes in mucosa of nasopharynx, $3.8 \%$ had thickened lateral and posterior wall of nasopharynx. Majority of nasal changes resolved after consevative management with antiallergic and nasal decongestant drops.

Table 4 - Findings of Diagnostic Laryngoscopy

\begin{tabular}{|l|c|c|}
\hline \multicolumn{1}{|c|}{ Findings on Laryngoscopy } & \multicolumn{2}{c|}{ Study Subjects } \\
\cline { 2 - 3 } & No. & $5.0 \%$ \\
\hline Post nasal discharge & 4 & $1.3 \%$ \\
\hline Hyperemia of vocal cord & 1 & $3.8 \%$ \\
\hline Swelling in interarytenoid region & 3 & $3.8 \%$ \\
\hline Edema of epiglottis & 3 & $1.3 \%$ \\
\hline $\begin{array}{l}\text { Mucous plug over ant or / and post } \\
\text { commissure }\end{array}$ & 1 & $85 \%$ \\
\hline No abnormality detected & 68 & \\
\hline
\end{tabular}

Table-4 shows that maximum (85\%) of study subjects were found with no abnormality on Diagnostic Laryngoscopy, $5 \%$ of study subjects had post nasal discharge, $3.8 \%$ subjects had edema of epiglottis and equal proportion had swelling in inter-arytenoid region. Only 1.3\% subjects had hyperaemia of vocal cords and equal number had growth of aryepiglottic folds. Another $1.3 \%$ subjects had mucus plug over anterior or posterior commissure.

\section{Table 5 - Findings on throat swab for AFB}

\begin{tabular}{|c|c|c|}
\hline \multirow{2}{*}{ Findings on throat swab } & \multicolumn{2}{|c|}{ Study Subjects } \\
\cline { 2 - 3 } & No. & $1.25 \%$ \\
\hline AFB Positive & 1 & $98.75 \%$ \\
\hline AFB Negative & 79 & \\
\hline
\end{tabular}

Table- 5 shows that on doing investigation of throat swab for AFB , 79 patients (98.75\%) of study subjects were AFB negative, and only one patient $(1.25 \%)$ of study subject was found to be AFB positive. In case of AFB positive subject, biopsy specimen was taken from lateral and posterior wall of nasopharyx where thickening and growth was found and sent for 
histopathological examination. On histopathological examination, it showed diffuse infiltration from lymphocytes, epitheloid granuloma, langhans giant cells with caseous necrosis. This confirmed the diagnosis of nasopharyngeal tuberculosis.

\section{DISCUSSION}

In India where tuberculosis is so prevalent, nasopharyngeal tuberculosis is not very common and isolated nasopharyngeal tuberculosis is still uncommon.

Cristina S Nieves4 study states that, the most common symptom of nasopharyngeal tuberculosis was cervical lymphadenopathy and other symptoms may include chronic cough, epistaxis, nasal obstruction or nasal discharge, but in our study nasopharyngeal tuberculosis case did not present with cervical lymphadenopathy but epistaxis, chronic cough, nasal discharge were present. So our study states that the patient of nasopharyngeal tuberculosis may present with varied clinical manifestations.

The study of Jay J rohwedder2 on upper respiratory tract tuberculosis states that, the primary tuberculosis of the larynx does not occur. Laryngoscopic appearance of tuberculous laryngitis makes it difficult to exclude from laryngeal carcinoma. Vocal cord paralysis and cervical lymphadenopathy commonly present in carcinoma larynx, but these were not seen among patients of tubercular laryngitis. So when patients comes with complains of weight loss, productive cough, hoarseness of voice, proper examination of chest roentgenogram and sputum smear will avoid diagnostic confusion between tubercular laryngitis and carcinoma larynx. The study of $E$ Tas et al6 on upper respiratory tract tuberculosis states that, in modern days, tuberculosis of the upper respiratory system is rarely seen because of introduction of effective chemotherapy. The majority of cases reported with nasopharyngeal tuberculosis have come from nations where tuberculosis is endemic.

The study of Ann D King5 on Magnetic Resonance Imaging on features of nasopharyngeal tuberculosis states that, there are two patterns of nasopharyngeal tuberculosis, the first pattern shows a discrete polypoidal mass in adenoids and the second pattern shows a diffuse mucosal thickening of one or two walls of nasopharynx. It also states that there are three main patterns of involvement on direct examination of the nasopharynx [1] normal nasopharynx [2] irregularity and ulceration of mucosa [3] mass lesion that may be bulging of the wall or a polypoid mass. in our study there was thickening and mucosal irregularity present on lateral and posterior wall of nasopharynx.

In our study, irregular mucosal thickening on lateral and posterior wall of nasopharynx was found on diagnostic nasal endoscopy in nasopharyngeal tuberculosis case. Study of Tashnin Rahman8 on diagnostic nasal endoscopy showed a smooth congested growth in the superior wall of nasopharynx.

In our study on histopathological examination of throat swab positive patient for AFB showed epitheloid granuloma with caseous necrosis. Similar findings also found on histopathological examination in the study ofTashnin Rahman8.

In our study it was observed that only one patient $(1.25 \%)$ of pulmonary tuberculosis developed nasopharyngeal tuberculosis. which was comparable with study of Tashnin Rahman8

The study of ETas et al 6 states that the diagnosis of upper respiratory tuberculosis made by [1] clinical picture [2] histological findings [3] response to anti-tubercular treatment. Above study also states that caseous granuloma was present in all upper respiratory tuberculosis cases. In our study we sent throat swab of all 
cases to detect acid fast bacilli, and it was found positive in only one case. So throat swab for detection of acid fast bacilli is a useful microbiological test. On histopathologic examination of positive case, epitheloid granuloma and caseous necrosis was found.

In our study the AFB positive nasopharyngeal tuberculosis case belongs to adult age group (28 years). Study of Chonticha Srivanitchapoom7 on nasopharyngeal tuberculosis states that, nasopharyngeal tuberculosis commonly occurs in adults and there are two peaks of frequencies, (1) between 15 to 30 years (2) between 50 to 60 years.

\section{CONCLUSION}

India is an endemic country for pulmonary tuberculosis specially Eastern U. P, due to high population density, low socio-economic status and lack of education. In our study out of 80 cases of pulmonary tuberculosis, only one case found with tuberculosis of upper respiratory tract along with pulmonary tuberculosis. Nasopharyngeal and laryngeal Tuberculosis mimic symptoms with carcinoma. Therefore, a complete clinical, radiological and histopathological examinations are required to confirm the diagnosis.

Being an otorhinolaryngologist we should always keep in mind the possibility of Tuberculosis in the differential diagnosis of nasopharyngeal/laryngeal mass. In this way our study is helpful to keep eye on the cases of upper respiratory tract tuberculosis among cases of pulmonary tuberculosis and increases the awareness about upper respiratory tract tuberculosis. Further study is needed with large sample size with long duration of treatment/follow up as these are few limitations in our study to make definitive conclusion.

\section{REFERENCES}

1. Global Tuberculosis Report, 2017. World Health Organization.
2. J.J. Rohwedder, "Upper respiratory tract tuberculosis, Sixteen cases in a general hospital" Annals of Internal Medicine. 1974; 80(6): pp. 708-713.

3. R.K. Mishra, B.K. Prasad \& Sunil Mathur, Nasopharyngeal tuberculosis. Med. J. Armed Forces,India. 2015;71(2):S586-S589.

4. Cristina S. Nieves, MD, Rubiliza DC. Onofre, MD, Fortuna Corazon A. Aberin-Roldan, MD, Rene Louie C. Gutierrez, MD. Nasopharyngeal Tuberculosis in a patient presenting with upper airway obstruction. Philippine Journal of Otolaryngology-Head and Neck Surgery. 2010; Vol.25(1).

5. King $A D$, Ahuja $A T$, Tse GM, van Hasselt $A C$, Chan AB. MR Imaging Features of Nasopharyngeal Tuberculosis: Report of Three Cases and Literature Review. American journal of Neuroradiology 2003;24(4):279-82.

6. ETas, E Sahin, S Vural, HTurkoz, A Gursel. Upper Respiratory Tract Tuberculosis: Our Experience Of Three Cases and Review Of Article. The International Journal of Otorhinolaryngology. 2006;6(1).

7. Srivanitchapoom, C., \&Sittitrai, P. (2016). Nasopharyngeal Tuberculosis: Epidemiology, Mechanism of Infection, Clinical Manifestations, and Management. International Journal of O t o I a ry ngology, $2016 ; 1-6$. doi:10.1155/2016/4817429.

8. Rahman T, llapakurty B, Kakati K, Das AK, Kataki AC. Primary nasopharyngeal tuberculosis: A rare case presentation. National Journal of Medical Research. 2016;6(2):224-5. 